Pacific Journal of Mathematics

LOCALIZATION OF A CERTAIN SUBGROUP OF
SELF-HOMOTOPY EQUIVALENCES 


\title{
LOCALIZATION OF A CERTAIN SUBGROUP OF SELF-HOMOTOPY EQUIVALENCES
}

\author{
KeN-IChi MARUYama
}

\begin{abstract}
Let $X$ be a simple, finite C.W. complex. The group $\mathscr{E}_{\#}(X)$ is known to be nilpotent. In this paper, we give a proof of the naturality of localization on this group, $\mathscr{E}_{\#}(X)_{(P)}=\mathscr{E}_{\#}\left(X_{(P)}\right)$. The result is then applied to study the group structures of $\mathscr{E}_{\#}(X)$ of rational Hopf spaces and some Lie groups.
\end{abstract}

Introduction. Let $X$ be a pointed topological space. We use the notation $\mathscr{E}(X)$ to denote the group of based self-homotopy equivalences of $X$. (For this group there are other notations, for example, $\operatorname{AUT}^{\circ}(X)$ in [2].) Throughout the paper our spaces $X$ will be connected of finite type and either finite dimensional or Postnikov pieces, namely, spaces with finite number of non-trivial homotopy groups. Then we denote by $\mathscr{E}_{\#}^{m}(X)$ the subgroup of $\mathscr{E}(X)$ which is the kernel of the obvious map (cf. [1], [16]):

$$
\mathscr{E}(X) \rightarrow \prod_{j \leq m} \operatorname{Aut} \pi_{j}(X) .
$$

We simply denote $\mathscr{E}_{\#}(X)$ when $m=\operatorname{dim} X$, where

$$
\operatorname{dim} X=\max \left\{i \mid \pi_{i}(X) \neq 0\right\}
$$

if $X$ is a Postnikov piece.

E. Dror and A. Zabrodsky have proved that $\mathscr{E}_{\#}(X)$ is a nilpotent group for an arbitrary finite dimensional C.W. complex or a Postnikov piece ([2], Theorem A). If $m \geq \operatorname{dim} X, \mathscr{E}_{\#}^{m}(X)$ is a subgroup of $\mathscr{E}_{\#}(X)$ and thus also nilpotent. Hence these groups can be localized in a natural way. For example, the reader may consult the book [5] which provides basic matters on the theory of localization of nilpotent groups (and spaces).

In this paper, our main result is the following.

TheOREM 0.1. Let $X$ be a simple C.W. complex and $P$ be an arbitrary collection of prime numbers. Assume that $m \geq \operatorname{dim} X$. Then the 
natural map:

$$
l_{\#:}: \mathscr{E}_{\#}^{m}(X) \rightarrow \mathscr{E}_{\#}^{m}\left(X_{(P)}\right)
$$

is the $P$-localization map, where $X_{(P)}$ is the localization at $P$.

In other words $\mathscr{E}_{\#}^{m}(X)_{(P)}=\mathscr{E}_{\#}^{m}\left(X_{(P)}\right)$. When a space is $P$-equivalent to the simpler space, this theorem enables us to determine $\mathscr{E}_{\#}(X)$ effectively. For example, for 0-regular spaces we obtain the following result which is concerned with the classical result of [1] (Theorem 5.4).

THEOREM 0.2. Let $X$ be a simple finite rational $H$-space with $\beta_{2 n_{1}-1}-$ $\operatorname{rank}_{Q}\left(\pi_{2 n_{i}-1}(X) \otimes Q\right) \leq 1$ for $i \leq k$. Then $\mathscr{E}_{\#}(X) /$ torsion $=Z \oplus \cdots \oplus Z$, the free abelian group of

$$
\operatorname{rank}=\sum_{i=1}^{k} \operatorname{rank}_{Q}\left(\pi_{2 n_{1}-1}(X) \otimes Q\right) \cdot\left(\beta_{2 n_{i}-1}-\operatorname{rank}_{Q}\left(\pi_{2 n_{i}-1}(X) \otimes Q\right)\right) \text {, }
$$

where $\beta_{j}$ is the jth Betti number and $H^{*}(X, Q)=E\left(x_{1}, \ldots, x_{k}\right)$ (the exterior algebra) with $\operatorname{deg} x_{i}=2 n_{i}-1$.

In many cases $\mathscr{E}_{\#}(X)$ is an abelian group. We give the following non-abelian example.

ExAmple (Example 3.1). $\mathscr{E}_{\#}(\mathrm{SO}(6))$ and $\mathscr{E}_{\#}(\mathrm{SU}(4))$ are not abelian.

This paper is organized as follows. In the first section we prove our main theorem (Theorem 0.1 ). In the second section we prove Theorem 0.2 . In the final section three we will show the above example.

1. Proof of the main theorem. Let $X_{n}$ be an $n$th Postnikov stage of $X$. Then there exist natural homomorphism $J_{X}^{n}: \mathscr{E}\left(X_{n}\right) \rightarrow \mathscr{E}\left(X_{n-1}\right)$ and its restriction, $J_{X}^{n}: \mathscr{E}_{\#}\left(X_{n}\right) \rightarrow \mathscr{E}_{\#}\left(X_{n-1}\right)$ which is denoted by the same symbol. When $m \geq \operatorname{dim} X, \mathscr{E}_{\#}^{m}(X)=\mathscr{E}_{\#}\left(X_{m}\right)$ and we can prove our theorem by induction on the Postnikov decomposition of $X$. The following exact sequence is due to Y. Nomura [10] (cf. [6], [13] and [16]).

$$
0 \rightarrow I\left(1_{X_{n}}\right) \rightarrow H^{n}\left(X_{n-1} ; \pi_{n}(X)\right) \stackrel{\Delta}{\longrightarrow} \mathscr{E}_{\#}\left(X_{n}\right) \stackrel{J_{X}^{n}}{\longrightarrow} \mathscr{E}_{\#}\left(X_{n-1}\right) .
$$

The localization map $l: X \rightarrow X_{(P)}$ can be restricted to the Postnikov stages and the following diagram is commutative.

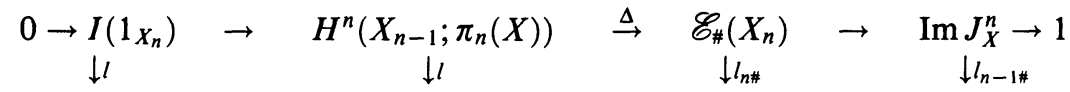

$$
\begin{aligned}
& 0 \rightarrow I\left(1_{X_{n(P)}}\right) \rightarrow H^{n}\left(X_{n-1(P)} ; \pi_{n}\left(X_{(P)}\right) \stackrel{\Delta}{\rightarrow} \mathscr{E}_{\#}\left(X_{n(P)}\right) \quad \rightarrow \quad \operatorname{Im} J_{X_{(P)}}^{n} \rightarrow 1\right. \text {. }
\end{aligned}
$$


In the above diagram,

$$
\begin{gathered}
l: H^{n}\left(X_{n-1} ; \pi_{n}(X)\right)=\left[X_{n-1}, K\left(\pi_{n}(X), n\right)\right] \rightarrow\left[X_{n-1}, K\left(\pi_{n}\left(X_{(P)}\right), n\right)\right] \\
=\left[X_{n-1(P)}, K\left(\pi_{n}\left(X_{(P)}\right), n\right)\right]=H^{n}\left(X_{n-1(P)} ; \pi_{n}\left(X_{(P)}\right)\right)
\end{gathered}
$$

obviously $P$-localizes. Since we can show by induction that $\mathscr{E}_{\#}\left(X_{n(P)}\right)$ is a $P$-local group for any $n$, it suffices to show that the restriction of $l_{n-1 \#}$ to $\operatorname{Im} J_{X}^{n}$ and the restriction of $l$ to $I\left(1_{X_{n}}\right)$ are both localization maps (Theorem 3.2 Ch. I, [5]).

(I) $l_{n-1 \#}: \operatorname{Im} J_{X}^{n} \rightarrow \operatorname{Im} J_{X_{(P)}}^{n} P$-localizes.

First we recall that $\operatorname{Im} J_{X}^{n}=\left\{f \in \mathscr{E}_{\#}\left(X_{n-1}\right) \mid f^{*} k^{n+1}=k^{n+1}\right\}$, where $k^{n+1}$ is the $k$-invariant of $X$ (cf. Theorem 2.9 [10]). This group can be identified with the isotropy subgroup at $k^{n+1}$ with respect to the action of $\mathscr{E}_{\#}\left(X_{n-1}\right)$ on the cohomology group $H^{n+1}\left(X_{n-1} ; \pi_{n}(X)\right)$.

Secondly we assert that this action is nilpotent (for the nilpotent action see $\S 4$ of [5]). Let us consider the fibrations

$$
K\left(\pi_{m}(X), m\right) \rightarrow X_{m} \rightarrow X_{m-1}, \quad m \leq n-1 .
$$

For $m=1, X_{1}=K\left(\pi_{1}(X), 1\right)$ and $\mathscr{E}_{\#}\left(X_{n-1}\right)$ acts trivially on the cohomology. There exists the $\mathscr{E}_{\#}\left(X_{n-1}\right)$-module spectral sequence converging to $H^{*}\left(X_{m}\right)$.

$$
E_{2}^{p, q}=H^{p}\left(X_{m-1} ; H^{q}\left(\pi_{m}(X), m\right)\right) \Rightarrow H^{*}\left(X_{m}\right) .
$$

If we assume that $\mathscr{E}_{\#}\left(X_{n-1}\right)$ acts on $H^{*}\left(X_{m-1}\right)$ nilpotently, then so does $H^{*}\left(X_{m}\right)$. Thus $\mathscr{E}_{\#}\left(X_{n-1}\right)$ acts on $H^{*}\left(X_{n-1}\right)$ nilpotently. By the universal coefficient theorem, we obtain the assertion.

Let $Q$ be a nilpotent group acting on a group $N$ nilpotently. Then the localizaton $Q_{P}$ acts on $N_{P}$ compatibly in the sense of $\S 1$ of [3].

Theorem. (P. Hilton [3], Theorem 1.1) $Q(a)_{P}=Q_{P}(e a)$, where $e: N \rightarrow N_{P}$ localizes and $Q(a)$ denotes the isotropy subgroup of $Q$ at $a \in N$.

By the hypothesis of induction, $l_{n-1 \#:}: \mathscr{E}_{\#}\left(X_{n-1}\right) \rightarrow \mathscr{E}_{\#}\left(X_{n-1(P)}\right)$ localizes and by the naturality of $l_{n-1 \#}$ it is compatible with the given action of $\mathscr{E}_{\#}\left(X_{n-1}\right)$ on the cohomology. Thus there is a commutative diagram:

$$
\begin{array}{ccc}
\mathscr{E}_{\#}\left(X_{n-1}\right) & \rightarrow & \text { Aut } H^{n+1}\left(X_{n-1} ; \pi_{n}(X)\right) \\
\downarrow l_{n-1 \#} & & \\
\mathscr{E}_{\#}\left(X_{n-1(P)}\right) & \rightarrow & \text { Aut } H^{n+1}\left(X_{n-1(P)} ; \pi_{n}\left(X_{P}\right)\right) .
\end{array}
$$


Finally put $Q=\mathscr{E}_{\#}\left(X_{n-1}\right), N=H^{n+1}\left(X_{n-1} ; \pi_{n}(X)\right)$ and $a=k^{n+1}$. Then (I) is derived from the above Hilton's Theorem.

(II) $l: I\left(1_{X_{n}}\right) \rightarrow I\left(1_{X_{n(P)}}\right) P$-localizes.

$I\left(1_{X_{n}}\right)$, the kernel of the homomorphism $\Delta$, is a subgroup of the group $H^{n}\left(X_{n-1} ; \pi_{n}(X)\right)$ and has the following form ([10]):

$$
I\left(1_{X_{n}}\right)=\left\{x \in H^{n}\left(X_{n-1} ; \pi_{n}(X)\right) \mid \mu\left(x \times 1_{X_{n}}\right) d=1_{X_{n}}\right\},
$$

where $\mu$ denotes the action of $K\left(\pi_{n}(X), n\right)$ on $X_{n}, d$ is a diagonal map. Hence $I\left(1_{X_{n}}\right)$ can be regarded as an isotropy subgroup at $1_{X_{n}}$ with respect to the action of $H^{n}\left(X_{n-1} ; \pi_{n}(X)\right)$ on $\left[X_{n}, X_{n}\right]$. In this case we cannot apply the argument like above because $\left[X_{n}, X_{n}\right]$ is not generally a group. But we can use the argument which is dual to that of the proof of Theorem 2.5, Ch. II, [5]. We have a fibration:

$$
X_{n} \stackrel{p_{n}}{\longrightarrow} X_{n-1} \stackrel{k^{n+1}}{\longrightarrow} K\left(\pi_{n}(X), n+1\right)(=K) .
$$

This gives rise to a fibration:

$$
F\left(X_{n}, X_{n}\right) \stackrel{p_{n *}}{\longrightarrow} F\left(X_{n}, X_{n-1}\right) \stackrel{k_{*}^{n+1}}{\longrightarrow} F\left(X_{n}, K\left(\pi_{n}(X), n+1\right)\right),
$$

where $F($, ) denotes the function space. Then we obtain the following commutative diagram

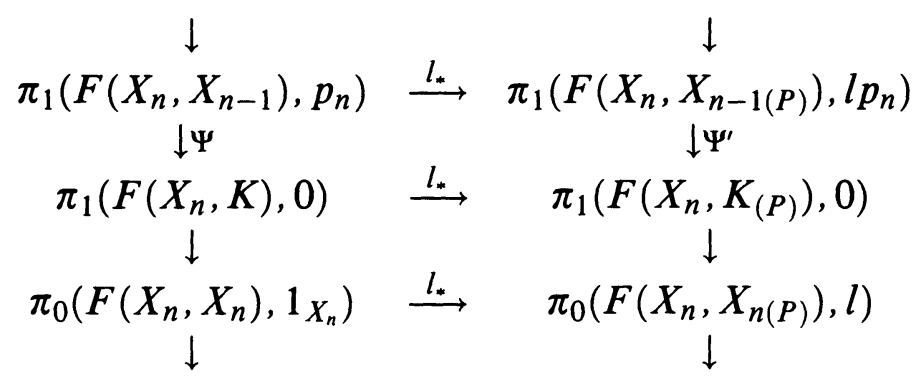

It is well known that $\operatorname{Im} \Psi\left(\operatorname{Im} \Psi^{\prime}\right)$ coincides with the isotropy subgroup of the $\pi_{1}\left(F\left(X_{n}, K\right), 0\right)=H^{n}\left(X_{n}, K\left(\pi_{n}(X), n\right)\right.$ action $\left(\pi_{1}\left(F\left(X_{n}, K_{(P)}\right), 0\right)=H^{n}\left(X_{n}, K\left(\pi_{n}\left(X_{(P)}\right), n\right)\right)\right.$ action $)$ on $\pi_{0}\left(F\left(X_{n}, X_{n}\right), 1_{X_{n}}\right)\left(\pi_{0}\left(F\left(X_{n}, X_{n(P)}\right), l\right)\right)$ at $1_{X_{n}}(l)$. We should note that these actions can be regarded as to be induced from the actions of $K\left(\pi_{n}(X), n\right)\left(K\left(\pi_{n}\left(X_{(P)}\right), n\right)\right)$ on $X_{n}\left(X_{n(P)}\right)$. Thus if we restrict these actions to $H^{n}\left(X_{n-1} ; \pi_{n}(X)\right)$ (or $H^{n}\left(X_{n-1} ; \pi_{n}\left(X_{(P)}\right)\right)$, we get actions mentioned earlier. Let $i_{N}: X_{n}^{N} \rightarrow X_{n}$ be the inclusion of $N$ skeleton of $X_{n}$, with $N$ sufficiently large. The space $F\left(X_{n}, X_{n-1}\right)=$ $F\left(\mathrm{lim}_{j} X_{n}^{N+j}, X_{n-1}\right)$ is homotopy equivalent to $\lim _{j} F\left(X_{n}^{N+j}, X_{n-1}\right)$. 
This gives rise to a homotopy equivalence of $\left(F\left(X_{n}, X_{n-1}\right), p_{n}\right)$ and $\lim _{j}\left(F\left(X_{n}^{N+j}, X_{n-1}\right), p_{n} i_{N+j}\right)$. We have a cofibration

$$
V \rightarrow X_{n}^{N+j} \rightarrow X_{n}^{N+j+1},
$$

where $V$ is a wedge of $N+j$-spheres, giving rise to a fibration

$$
\left(F\left(V, X_{n-1}\right), 0\right) \leftarrow\left(F\left(X_{n}^{N+j}, X_{n-1}\right), p_{n} i_{N+j}\right) \leftarrow\left(F\left(X_{n}^{N+j+1}, X_{n-1}\right), p_{n} i_{N+j+1}\right) .
$$

Since $N$ is sufficiently large, $\left(F\left(V, X_{n-1}\right), 0\right)$ is weakly contractible, thus the total space and fibre of this fibration are weakly homotopy equivalent. As a result,

$$
\begin{aligned}
\left(F\left(X_{n}, X_{n-1}\right), p_{n}\right) & =\underset{\lim }{\leftrightarrows}\left(F\left(X_{n}^{N+j}, X_{n-1}\right), p_{n} i_{N+j}\right) \\
& \simeq\left(F\left(X_{n}^{N}, X_{n-1}\right), p_{n} i_{N}\right) .
\end{aligned}
$$

Thus these are nilpotent spaces by Theorem 2.5, Ch. II, [5] and moreover the upper $l_{*}$ in the above diagram localizes (Theorem 3.11, Ch. II, [5]) and so does the middle $l_{*}$. Therefore, $l_{*}: \operatorname{Im} \Psi \rightarrow \operatorname{Im} \Psi^{\prime}$ localizes. We have the following.

$$
\begin{aligned}
I\left(1_{X_{n}}\right)_{(P)} & =\left(\operatorname{Im} \Psi \cap H^{n}\left(X_{n-1} ; \pi_{n}(X)\right)_{(P)}\right. \\
& =(\operatorname{Im} \Psi)_{(P)} \cap H^{n}\left(X_{n-1} ; \pi_{n}(X)_{(P)}\right), \quad \text { by Theorem 1.2, [4] } \\
& =\operatorname{Im} \Psi^{\prime} \cap H^{n}\left(X_{n-1(P)} ; \pi_{n}\left(X_{(P)}\right)\right) \\
& =I\left(1_{\left.X_{n(P)}\right)}\right)
\end{aligned}
$$

This completes the proof of (II).

2. Proof of Theorem 0.2. In this section we prove Theorem 0.2 . Again we use the induction on the Postnikov decomposition. First we introduce the following proposition.

Proposition 2.1. Let $X=K\left(Q, 2 n_{k}-1\right) \times \cdots \times K\left(Q, 2 n_{1}-1\right)$, $1 \leq n_{1} \leq \cdots \leq n_{k}$ with $\beta_{2 n_{i}-1}-\operatorname{rank}_{Q}\left(\pi_{2 n_{i}-1}(X)\right) \leq 1$ for $i \leq k$. Then $\mathscr{E}_{\#}(X)=Q \oplus \cdots \oplus Q$, the direct sum of rationals of the rank (over $Q)=\sum_{l=1}^{k} \operatorname{rank}_{Q}\left(\pi_{2 n_{l}-1}(X)\right) \cdot\left(\beta_{2 n_{l}-1}-\operatorname{rank}_{Q}\left(\pi_{2 n_{l}-1}(X)\right)\right)$.

Proof. On the first Postnikov stage, $\mathscr{E}_{\#}\left(X_{2 n_{1}-1}\right)=1$. Assume that $\mathscr{E}_{\#}\left(X_{2 n_{l-1}-1}\right)$ is an abelian group. By Theorem 2.10 of [11],

$$
\mathscr{E}_{\#}\left(X_{2 n_{l}-1}\right)=H^{2 n_{l}-1}\left(X_{2 n_{l-1}-1} ; \pi_{2 n_{l}-1}(X)\right) \underset{T}{\times} E_{\#}\left(X_{2 n_{l}-1}\right),
$$


where $\times$ means the semidirect product. Moreover the action of $f$ on $\omega\left(\stackrel{T}{f} \in \mathscr{E}_{\#}\left(X_{2 n_{l-1}-1}\right), \omega \in H^{2 n_{l}-1}\left(X_{2 n_{l-1}-1} ; \pi_{2 n_{l}-1}(X)\right)\right)$ is given by $f \cdot \omega=(f)^{*-1} \omega$. Each element $\omega$ can be written as the sum,

$$
\sum a_{n_{\ell(1)}} \cup \cdots \cup a_{\left.n_{\ell(m)}\right)},
$$

where $a_{n_{i(j)}}$ is the fundamental class of $H^{2 n_{i(j)}}\left(K\left(Q, 2 n_{i(j)}-1\right) ; Q\right)$, $1 \leq i(j) \leq k, \cup$ means the cup product. Obviously, $(f)^{*-1}$ maps $\omega$ into $\omega$ identically. It follows that $f \cdot \omega=\omega$. What we have just proved is that $\mathscr{E}_{\#}\left(X_{2 n_{l}-1}\right)$ is actually the direct product of $\mathscr{E}_{\#}\left(X_{2 n_{l-1}-1}\right)$ and the cohomology group. The rank (over $Q$ ) of $\mathscr{E}_{\#}(X)$ can be computed as follows (cf. the proof of Theorem 5.4 [1]). Let $\rho_{l}$ stand for the dimension of $\mathscr{E}_{\#}\left(X_{n_{1}-1}\right)$.

$$
\rho_{l}=\operatorname{rank}_{Q}\left(H^{2 n_{l}-1}\left(X_{2 n_{l-1}-1} ; \pi_{2 n_{l}-1}(X)\right)\right)+\rho_{l-1} .
$$

Therefore,

$$
\begin{aligned}
\operatorname{rank}_{Q} \mathscr{E}_{\#}(X) & =\rho_{k}=\sum_{l=2}^{k} \operatorname{rank}_{Q}\left(H^{2 n_{l}-1}\left(X_{2 n_{l-1}-1} ; \pi_{2 n_{l}-1}(X)\right)\right) \\
& =\sum_{l=1}^{k} \operatorname{rank}_{Q}\left(\pi_{2 n_{l}-1}(X)\right) \cdot\left(\beta_{2 n_{l}-1}-\operatorname{rank}_{Q}\left(\pi_{2 n_{l}-1}(X)\right)\right) .
\end{aligned}
$$

Proof of the theorem. By the above proposition, $\mathscr{E}_{\#}\left(X_{0}\right)=$ the direct sum of rationals. Thus $\mathscr{E}_{\#}(X)_{(0)}=\mathscr{E}_{\#}\left(X_{(0)}\right)$ is also an abelian group. Recall that all torsion elements of a nilpotent subgroup form a normal subgroup. Then the injectivity of rationalization,

$$
\mathscr{E}_{\#}(X) / \text { torsion } \rightarrow\left(\mathscr{E}_{\#}(X) / \text { torsion }\right)_{(0)}=\mathscr{E}_{\#}(X)_{(0)},
$$

implies that $\mathscr{E}_{\#}(X) /$ torsion is a free abelian group of the rank mentioned above (since we are assuming that $X$ is of finite type, this group is finitely genrated).

\section{Further application.}

EXAMPLE 3.1 Let $\Pi$ be the collection of all primes. We see that $\mathscr{E}_{\#}(\mathrm{SO}(6))_{(\Pi-2)}=\mathscr{E}_{\#}(\mathrm{SU}(4))_{(\Pi-2)}$. Let us denote this group by $G$. Then $G=G_{(3)} \times Z_{5} \times Z_{5}$ and $G_{(3)}$ has the following presentation (cf. [11]).

$$
G_{(3)}=\left\langle a, b, c \mid a^{9}, b^{9}, c^{3},[a, b],[a, c],[b, c] a^{-3}\right\rangle \text {. }
$$

Proof. $\mathrm{SO}(6) \simeq_{p} \mathrm{Spin}(6)=\mathrm{SU}(4)$ for an odd prime $p$, where $\simeq_{p}$ denotes the $p$-equivalence. Due to this equivalence a half part of the 
Example 3.1 is obvious. To determine the group structure we recall the following theorem.

Theorem (Sieradski, Theorem 4, Corollary 8, [14]). Let $X$ and $Y$ be homotopy associative $H$-spaces. If the homotopy set $[A \vee B, A \wedge B]$ is trivial, then there is a short exact sequence of multiplicative groups.

$$
1 \rightarrow[X \wedge Y, X \times Y] \stackrel{q^{*}+1}{\longrightarrow} \mathscr{E}(X \times Y) \rightarrow \mathrm{GL}\left(2, \Lambda_{I J}\right) \rightarrow 1,
$$

where $q: X \times Y \rightarrow X \wedge Y$ is the projection, for $\Lambda_{I J}$ see [14].

The group structure. Let us denote $\Pi-2$ by $l$. There is another l-equivalence: $\mathrm{SO}(6) \simeq_{l} \mathrm{SO}(5) \times S^{5}$. We first investigate the group $\mathscr{E}\left(\mathrm{SO}(5)_{(l)} \times S_{(l)}^{5}\right)$. As it is well known, $\mathrm{SO}(5)_{(l)}$ is homotopy equivalent to $\operatorname{Sp}(2)_{(l)}$. $\left(\operatorname{SO}(5) \wedge S^{5}\right)_{(l)} \simeq\left(\operatorname{Sp}(2) \wedge S^{5}\right)_{(l)} \simeq\left(S^{8} \cup e^{12} \vee S^{15}\right)_{(l)}$, the triviality of the attaching map of the top cell is due to Lemma 2.1 , (ii), [7]. We use this cell structure to obtain (i) $\left[\left(\mathrm{SO}(5) \vee S^{5}\right)_{(l)}\right.$, $\left.\left(\mathrm{SO}(5) \wedge S^{5}\right)_{(l)}\right]=0$, (ii) $\left[\mathrm{SO}(5)_{(l)}, S_{(l)}^{5}\right]=0=\left[S_{(l)}^{5}, \mathrm{SO}(5)_{(l)}\right]$. Therefore (3.2) $\left(X=\mathrm{SO}(5)_{(l)}, Y=S_{(l)}^{5}\right)$, its restriction, has the following form.

$$
0 \rightarrow\left[\left(S^{8} \cup e^{12}\right) \vee S^{15}, \mathrm{Sp}(2)_{(l)} \times S_{(l)}^{5}\right] \rightarrow \mathscr{E}_{\#}\left(\mathrm{SO}(6)_{(l)}\right) \rightarrow \mathscr{E}_{\#}^{(15)}(\mathrm{Sp}(2))_{(l)} \rightarrow 1 .
$$

The left-hand side term of this sequence is isomorphic to $Z_{9} \oplus Z_{45}$. Let us consider the 3-component. First we recall the result of [12]. Let $\lambda$ be the map which is introduced in [12] $(\S 1,1.2)$ :

$$
\lambda: \pi_{10}(\operatorname{Sp}(2)) \rightarrow \mathscr{E}(\operatorname{Sp}(2)) .
$$

Then a generator of $\mathscr{E}_{\#}(\operatorname{Sp}(2))_{(3)}\left(=\mathscr{E}_{\#}(\operatorname{SO}(5))_{(3)}\right)=Z_{3}$ can be represented by $\lambda\left(i \alpha_{2}\right)$, where $i: S^{3} \rightarrow \mathrm{Sp}(2)$ is the inclusion and $\alpha_{2} \in$ $\pi_{10}\left(S^{3}\right)_{(3)}=Z_{3}$ is the generator [15]. Using the group structure of $\mathrm{Sp}(2), \lambda\left(i \alpha_{2}\right)$ has the other description $1+i \alpha_{2} p$ (Corollary 2.2, [8]), where $p$ is the collapsing map to the top cell. The only nontrivial homotopy group $\pi_{i}(\operatorname{Sp}(2))_{(3)}, 10<i \leq 15$, is $\pi_{14}(\operatorname{Sp}(2))_{(3)}=Z_{3}$ and its generator is $i \alpha_{3}(3)$ [9], where $\alpha_{3}(3) \in \pi_{14}\left(S^{3}\right)_{(3)}=Z_{3}$, is the generator. Since $\left(1+i \alpha_{2} p\right)\left(i \alpha_{3}(3)\right)=i \alpha_{3}(3)$ it follows that $\mathscr{E}_{\#}^{15}(\operatorname{Sp}(2))_{(3)}=$ $\mathscr{E}_{\#}(\operatorname{Sp}(2))_{(3)}$. Similarly, $\mathscr{E}_{\#}^{15}(\operatorname{Sp}(2))_{(5)}=\mathscr{E}_{\#}(\operatorname{Sp}(2))_{(5)}$.

A generator of the summand $Z_{9}=\left(Z_{45}\right)_{(3)}$ on the left-hand side of (3.3) may be given by:

$$
S^{8} \cup e^{12} \vee S^{15} \stackrel{r}{\longrightarrow} S^{8} \cup e^{12} \stackrel{\tilde{\alpha}_{1}}{\longrightarrow} S^{5} \stackrel{j}{\longrightarrow} \mathrm{SO}(5) \times S^{5},
$$


where $r$ is the retraction and $\tilde{\alpha}_{1}$ stands for the extension of $\alpha_{1} \in$ $\pi_{8}\left(S^{5}\right)_{(3)}=Z_{3}$ is the generator, $j$ is the natural inclusion. The composition,

$$
\left(\lambda\left(-\alpha_{2}\right) \times 1\right)\left(1+q^{\#}\left(j \tilde{\alpha}_{1} r\right)\right)\left(\lambda\left(\alpha_{2}\right) \times 1\right) \in \mathscr{E}\left(\operatorname{Sp}(2) \times S^{5}\right)
$$

is homotopic to $\left(\lambda\left(-\alpha_{2}\right) \times 1\right)\left(\lambda\left(\alpha_{2}\right) \times 1+\left(j \tilde{\alpha}_{1} r\right) q\left(\lambda\left(\alpha_{2}\right) \times 1\right)\right)$.

$\left(j \tilde{\alpha}_{1} r\right) q\left(\lambda\left(\alpha_{2}\right) \times 1\right)$ can be easily seen to be $\left(j \tilde{\alpha}_{1} r\right)\left(\lambda\left(\alpha_{2}\right) \wedge 1\right)$. By the definition of $\lambda, \lambda\left(\alpha_{2}\right) \wedge 1$ is homotopic to $1+\Sigma^{5}\left(i \alpha_{2} p\right)$. Hence $\left(j \tilde{\alpha}_{1} r\right)\left(\lambda\left(\alpha_{2}\right) \wedge 1\right)=j \tilde{\alpha}_{1} r+j \alpha_{1} \Sigma^{5}\left(\alpha_{2} p\right)$. Hence (3.4) is equal to $1+$ $q^{\#}\left(j \tilde{\alpha}_{1} r+j \alpha_{1} \Sigma^{5}\left(\alpha_{2} p\right)\right)$. At $\pi_{15}\left(S^{5}\right), \alpha_{1} \alpha_{2}=-3 \beta_{1}(5)[15]$, p. 180 . The analogous argument permits us to show that the $\left(1+q^{\#}\right)$-image of the other $Z_{9}$-summand commutes with $\lambda\left(\alpha_{2}\right) \times 1$.

Put $c=\lambda\left(-\alpha_{2}\right) \times 1, b=1+q^{\#}\left(j\left(-\tilde{\alpha}_{1}(r)\right), a=1+q^{\#} j \beta_{1}(5)\left(\Sigma^{5} p\right)\right.$. These imply the assertion on the group structure of $G_{(3)}$. Since we see easily that the 5-components have no non-trivial extensions, we complete the proof.

\section{REFERENCES}

[1] M. Arkowitz and C. R. Curjel, Groups of homotopy classes, Lecture Notes in Math., 4 (1967).

[2] E. Dror and A. Zabrodsky, Unipotency and nilpotency in homotopy equivalences, Topology, 18 (1979), 187-197.

[3] P. Hilton, On orbit set for group actions and localization, Lecture Notes in Math., 673 (1978), 185-201.

[4] , Nilpotent actions on nilpotent groups, Proc. Logic and Math. Conference, Lecture Notes in Math., 450 (1975), 174-196.

[5] P. Hilton, G. Mislin and J. Roitberg, Localization of Nilpotent Groups and Spaces, Mathematics Studies, 15 (1975), North Holland.

[6] D. W. Kahn, The group of homotopy equivalences, Math. Z., 84 (1964), 1-8.

[7] M. Mimura, The number of multiplications on $\mathrm{SU}(3)$ and $\mathrm{Sp}(2)$, Trans. Amer. Math. Soc., 146 (1969), 473-492.

[8] M. Mimura and N. Sawashita, On the group of self-homotopy equivalences of $H$-spaces of rank 2, J. Math. Kyoto Univ., 21 (1981), 331-349.

[9] M. Mimura and H. Toda, Homotopy groups of $\mathrm{SU}(3), \mathrm{SU}(4)$ and $\mathrm{Sp}(2)$, J. Math. Kyoto Univ., 3 (1964), 251-273.

[10] Y. Nomura, Homotopy equivalences in a principal fibre space, Math. Z., 92 (1966), 380-388.

[11] S. Oka, On the group of self homotopy equivalences of $H$-spaces of low rank II. Mem. Fac. Sci., Kyushu Univ., Ser A, 35 (1981), 307-323.

[12] S. Oka, N. Sawashita and M. Sugawara, On the group of self-equivalences of a mapping cone, Hiroshima Math. J., 4 (1974), 9-28.

[13] J. W. Rutter, Groups of self homotopy equivalences of induced spaces, Comment. Math. Helv., 45 (1970), 236-255.

[14] A. J. Sieradski, Twisted self-homotopy equivalences, Pacific J. Math., 34 (1970), 789-802. 
[15] H. Toda, Composition methods in homotopy groups, Ann. of Math. Studies., 49 (1962), Princeton Univ. Press.

[16] K. Tsukiyama, Self-homotopy-equivalences of a space with two nonvanishing homotopy groups, Proc. Amer. Math. Soc., 79 (1980), 134-138.

Received October 15, 1987 and in revised form April 15, 1988.

KYUSHU UNIVERSITY

HAKOZAKI FUKUOKA 812

JAPAN

Current address: Department of Mathematics

Faculty of Education

Chiba University

Yayoicho, Chiba 260

Japan 



\section{PACIFIC JOURNAL OF MATHEMATICS \\ EDITORS}

\author{
V. S. VARADARAJAN \\ (Managing Editor) \\ University of California \\ Los Angeles, CA 90024 \\ HERBERT ClEMENS \\ University of Utah \\ Salt Lake City, UT 84112 \\ THOMAS ENRIGHT \\ University of California, San Diego \\ La Jolla, CA 92093
}

R. FINN

Stanford University

Stanford, CA 94305

HERMANN FLASCHKA

University of Arizona

Tucson, AZ 85721

VAUGhan F. R. JONES

University of California

Berkeley, CA 94720

STEVEN KERCKHOFF

Stanford University

Stanford, CA 94305

\section{ROBION KIRBY}

University of California

Berkeley, CA 94720

C. C. MOORE

University of California

Berkeley, CA 94720

HAROLD STARK

University of California, San Diego

La Jolla, CA 92093

\section{ASSOCIATE EDITORS}
R. ARENS
E. F. BECKENBACH
B. H. NEUMANN
F. WOLF
K. YOSHIDA

(1906-1982)

\section{SUPPORTING INSTITUTIONS}

UNIVERSITY OF ARIZONA

UNIVERSITY OF BRITISH COLUMBIA

CALIFORNIA INSTITUTE OF TECHNOLOGY

UNIVERSITY OF CALIFORNIA

MONTANA STATE UNIVERSITY

UNIVERSITY OF NEVADA, RENO

NEW MEXICO STATE UNIVERSITY

OREGON STATE UNIVERSITY

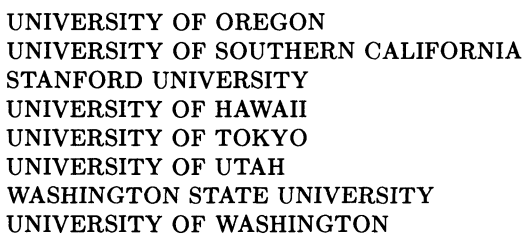

The Supporting Institutions listed above contribute to the cost of publication of this Journal, but they are not owners or publishers and have no responsibility for its content or policies.

\footnotetext{
Mathematical papers intended for publication in the Pacific Journal of Mathematics should be in typed form or offset-reproduced (not dittoed), double spaced with large margins. Please do not use built up fractions in the text of the manuscript. However, you may use them in the displayed equations. Underline Greek letters in red, German in green, and script in blue. The first paragraph must be capable of being used separately as a synopsis of the entire paper. In particular it should contain no bibliographic references. Please propose a heading for the odd numbered pages of less than 35 characters. Manuscripts, in triplicate, may be sent to any one of the editors. Please classify according to the scheme of Math. Reviews, Index to Vol. 39. Supply name and address of author to whom proofs should be sent. All other communications should be addressed to the managing editor, or Elaine Barth, University of California, Los Angeles, California 90024.

There are page-charges associated with articles appearing in the Pacific Journal of Mathematics. These charges are expected to be paid by the author's University, Government Agency or Company. If the author or authors do not have access to such Institutional support these charges are waived. Single authors will receive 50 free reprints; joint authors will receive a total of 100 free reprints. Additional copies may be obtained at cost in multiples of 50 .
}

The Pacific Journal of Mathematics is issued monthly as of January 1966. Regular subscription rate: $\$ 190.00$ a year (5 Vols., 10 issues). Special rate: $\$ 95.00$ a year to individual members of supporting institutions.

Subscriptions, orders for numbers issued in the last three calendar years, and changes of address should be sent to Pacific Journal of Mathematics, P.O. Box 969, Carmel Valley, CA 93924, U.S.A. Old back numbers obtainable from Kraus Periodicals Co., Route 100, Millwood, NY 10546.

The Pacific Journal of Mathematics at P.O. Box 969, Carmel Valley, CA 93924 (ISSN 0030-8730) publishes 5 volumes per year. Application to mail at Second-class postage rates is pending at Carmel Valley, California, and additional mailing offices. Postmaster: send address changes to Pacific Journal of Mathematics, P.O. Box 969, Carmel Valley, CA 93924.

PUBLISHED BY PACIFIC JOURNAL OF MATHEMATICS, A NON-PROFIT CORPORATION Copyright (C) 1989 by Pacific Journal of Mathematics 


\section{Pacific Journal of Mathematics}

\section{Vol. 136, No. 2 December, 1989}

A. K. Agarwal and David Bressoud, Lattice paths and multiple basic hypergeometric series ................................. 209

Adalberto Panobianco Bergamasco and Hermano de Souza Ribeiro, Uniqueness in a doubly characteristic Cauchy problem ........... 229

Thomas Curtis Craven and George Leslie Csordas, Jensen polynomials

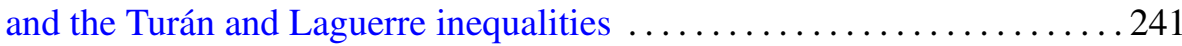

Gary R. Jensen and Marco Rigoli, Harmonic Gauss maps ............ 261

L. G. Kovács and Cheryl Elisabeth Praeger, Finite permutation groups with large abelian quotients $\ldots \ldots \ldots \ldots \ldots \ldots \ldots \ldots \ldots \ldots \ldots \ldots . \ldots \ldots$

Ken-ichi Maruyama, Localization of a certain subgroup of self-homotopy

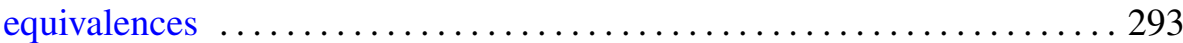

Tomasz Mazur, Canonical isometry on weighted Bergman spaces ......... 303

Bernt Karsten Oksendal, A stochastic Fatou theorem for quasiregular

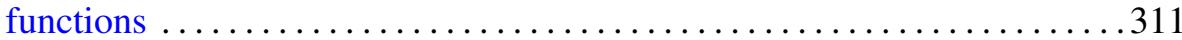

Ian Fraser Putnam, The $C^{*}$-algebras associated with minimal homeomorphisms of the Cantor set ........................ 329

Tom Joseph Taylor, Some aspects of differential geometry associated with

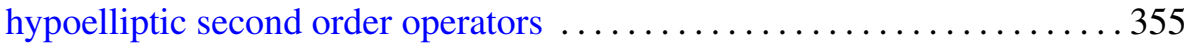

Tom Joseph Taylor, Off diagonal asymptotics of hypoelliptic diffusion equations and singular Riemannian geometry 